\title{
Quasi-normal mode expansion as a tool for the design of nanophotonic devices
}

\author{
Rémi Colom ${ }^{1, *}$, Felix Binkowski ${ }^{1}$, Fridtjof Betz ${ }^{1}$, Martin Hammerschmidt ${ }^{2}$, Lin Zschiedrich ${ }^{2}$, and Sven Burger ${ }^{1,2, * *}$ \\ ${ }^{1}$ Zuse Institute Berlin, Takustraße 7, 14195 Berlin, Germany \\ ${ }^{2} \mathrm{JCM}$ wave GmbH, Bolivarallee 22, 14050 Berlin, Germany
}

\begin{abstract}
Many nanophotonic devices rely on the excitation of photonic resonances to enhance light-matter interaction. The understanding of the resonances is therefore of a key importance to facilitate the design of such devices. These resonances may be analyzed by use of the quasi-normal mode (QNM) theory. Here, we illustrate how QNM analysis may help study and design resonant nanophotonic devices. We will in particular use the QNM expansion of far-field quantities based on Riesz projection to design optical antennas.
\end{abstract}

\section{Introduction}

Nanophotonic devices allow to control and tailor lightmatter interactions at the nanoscale. Many applications such as quantum infomation technology, bio-sensing or the use of metasurfaces as miniature optical components may benefit from the use of nanophotonic devices. This control of light-matter interactions is often achieved through the excitation of the photonic resonances of these nanophotonic devices. The resonant interaction between light and nanometric structures may readily be described by using QNM theory $[1,2]$. The design of nanophotonic devices could consequently be facilitated by using QNM analysis. QNM are however diverging with the distance from the resonator. This complicates the analysis of farfield quantities using QNM $[1,2]$. Here, we will use QNM expansion of far-field quantities based on Riesz projection [4] to understand and design nanophotonic devices. We will in particular focus on the design of optical antennas.

\section{Design of optical antennas using quasi-normal mode expansions}

When designing nanophotonic devices, it is usually necessary to shape and control both the far-field and near-field properties of photonic structures. This may necessitate the careful tuning of many parameters or degrees of freedom of the structure under consideration and consequently result in time-consuming and costly parameter scanning. Even if this design may be assisted by using optimization approaches [5, 6], it would still be valuable to fully characterize the optical properties of a photonic structure in terms of a few number of modes. These modes are solution of the source-free Maxwell's equations:

$$
\nabla \times \mu_{0}^{-1} \nabla \times \mathbf{E}_{k}(\mathbf{r})-\omega_{k}^{2} \varepsilon\left(\mathbf{r}, \omega_{k}\right) \mathbf{E}_{k}(\mathbf{r})=0
$$

with outgoing boundary conditions. The eigenfrequencies $\omega_{k}$ are in general complex valued.

The design of optical antennas is an example where one needs to control both the near-field and far-field optical behavior of photonic structures. Tuning the near-field properties of the photonic stucture allows one to have a control over the Purcell factor enhancement $\Gamma(\omega)$ :

$$
\Gamma(\omega)=-\frac{\operatorname{Re}\left(\mathbf{E}\left(\mathbf{r}_{d}, \omega\right) \cdot \mathbf{j}^{*}\right)}{2 \Gamma_{b}}
$$

$\mathbf{j}=-i \omega \mathbf{p}, \mathbf{p}$ being the dipole moment while $\Gamma_{b}$ is the dipole emission in the homogeneous background material and $\mathbf{r}_{d}$ is the position of the dipole. This quantity may be expanded on the QNM basis by means of the Riesz projection $\Gamma(\omega)=\sum_{k=1}^{k_{\max }} \tilde{\Gamma}_{k}(\omega)+\Gamma_{r}(\omega)$ where $\tilde{\Gamma}_{k}(\omega)$ are the resonant contribution and $\Gamma_{r}(\omega)$ is the non resonant background contribution which are defined as follows [3]:

$$
\begin{aligned}
& \tilde{\Gamma}_{k}(\omega)=\frac{1}{2 \Gamma_{b}} \operatorname{Re}\left[-\frac{1}{2 i \pi}\left(\oint_{C_{k}} \frac{\mathbf{E}\left(\mathbf{r}_{d}, z\right)}{z-\omega} d z\right) \cdot \mathbf{j}^{*}\right] \\
& \Gamma_{r}(\omega)=\frac{1}{2 \Gamma_{b}} \operatorname{Re}\left[\frac{1}{2 i \pi}\left(\oint_{C_{r}} \frac{\mathbf{E}\left(\mathbf{r}_{d}, z\right)}{z-\omega} d z\right) \cdot \mathbf{j}^{*}\right]
\end{aligned}
$$

where the contours $C_{k}$ are closed contours around the eigenfrequencies $\omega_{k}$ while the contour $C_{r}$ is a large contour permitting to take into account the non-resonant background. One sees that the Purcell factor can thus be described in terms of a small number of resonant and nonresonant terms. One can then vary the opto-geometrical parameter of a photonic sctructure to tune the peak position and width of resonant contributions $\tilde{\Gamma}_{k}(\omega)$ in order to optimize the Purcell factor for a given application.

Besides the near-field behavior, the far-field properties of optical antennas are also of particular interest. Optical antennas can indeed allow to control the radiation pattern of a quantum emitter. This is of particular importance for certain applications where the light emitted has then to be 
collected by a lens or an optical fiber with a given numerical aperture $[6,7]$. It would then be very useful to describe the far-field response of these photonic structures in terms of their modes. In particular, a modal expansion of the Poynting vector would be of a special interest. This is however quite challenging since quasi-normal modes are exponentially diverging with the distance from the resonator $[1,2,4]$. To circumvent this divergence problem, we developed a method for expanding quadratic quantities and in particular the Poynting vector $\mathbf{S}(\mathbf{r}, \omega)=$ $s\left(\mathbf{E}(\mathbf{r}, \omega), \mathbf{E}^{*}(\mathbf{r}, \omega)\right)=\frac{1}{2} \operatorname{Re}\left(\mathbf{E}^{*}(\mathbf{r}, \omega) \times \frac{1}{i \omega \mu_{0}} \nabla \times \mathbf{E}(\mathbf{r}, \omega)\right)$. We found that the Poynting vector admits the following modal expansion [4]:

$$
\begin{aligned}
\mathbf{S}(\mathbf{r}, \omega)= & -\sum_{k=1}^{k_{\max }} \frac{1}{2 i \pi} \oint_{C_{k}} \frac{s\left(\mathbf{E}(\mathbf{r}, z), \mathbf{E}^{\circ}(\mathbf{r}, z)\right)}{z-\omega} d z \\
& -\sum_{k=1}^{k_{\max }} \frac{1}{2 i \pi} \oint_{C_{k}^{*}} \frac{s\left(\mathbf{E}(\mathbf{r}, z), \mathbf{E}^{\circ}(\mathbf{r}, z)\right)}{z-\omega} d z \\
& +\frac{1}{2 i \pi} \oint_{C_{r}} \frac{s\left(\mathbf{E}(\mathbf{r}, z), \mathbf{E}^{\circ}(\mathbf{r}, z)\right)}{z-\omega} d z
\end{aligned}
$$

where $\mathbf{E}^{\circ}(\mathbf{r}, z)$ is defined as the analytic continuation of $\mathbf{E}(\mathbf{r},-\omega)$ to the entire complex plane. The contour $C_{k}$ and $C_{r}$ are analogous to the contours used for the expansion of the Purcell factor in Eq. (3) while $C_{k}^{*}$ are the contours around the resonance poles of $\mathbf{E}^{\circ}(\mathbf{r}, z)$ equal to $\omega_{k}^{*}$. The important point is that the expansion provided in Eq. (4) is not diverging as $|\mathbf{r}|$ increases. This expansion can thus be used to study the far-field properties of photonic resonators. One can in particular describe the emission pattern in terms of resonant and non-resonant contributions. This can help shape the emission pattern by tuning the shape of the photonic structure under consideration to selectively excite the modes allowing to obtain the desirable emission pattern.
We will illustrate the usefulness of QNM expansion to design several types of optical antenna. We will start by presenting results on the circular Bragg gratings [4, 7]. We will also show how QNM expansion can used to understand the structures supporting quasi-bound states in the continuum originating from the avoided crossing of two resonances $[8,9]$.

\section{Acknowledgement}

We acknowledge funding by the Deutsche Forschungsgemeinschaft (DFG, German Research Foundation) under Germany's Excellence Strategy - The Berlin Mathematics Research Center MATH+(EXC-2046/1, project ID: 390685689, AA4-6). We acknowledge the Helmholtz Association for funding within the Helmholtz Excellence Network SOLARMATH, a strategic collaboration of the DFG Excellence Cluster MATH+and Helmholtz-Zentrum Berlin (grant no. ExNet-0042-Phase-2-3)

\section{References}

[1] P. Lalanne et al., Laser and Photonics Reviews, 12, 1700113 (2018).

[2] P. Kristensen et al., arXiv:1910.05412, (2019).

[3] L. Zschiedrich et al., Physical Review A, 98, 043806 (2018).

[4] F. Binkowski et al., arXiv:2003.11305, (2020).

[5] P. Schneider et al., ACS Photonics, 6, 2726-2733 (2019).

[6] P. Schneider et al., Optics Express, 26, 8479-8492 (2018).

[7] L. Rickert et al., Optics Express, 27, 36824-36837 (2019).

[8] M. Rybin et al., Physical Review Letters, 119, 243901 (2017).

[9] K. Koshelev et al., Science, 367, 288-292 (2020). 